\title{
THE EFFECTIVENESS OF ACCEPTANCE AND COMMITMENT THERAPY ON ANXIETY REDUCTION IN CANCER PATIENTS: META-ANALYSIS
}

\author{
Prajna Pramitha Purba
}

\author{
Masters Program in Public Health, Universitas Sebelas Maret
}

\begin{abstract}
Background: Cancer is a life threatening disease, the diagnosis and treatment of which can be very disruptive in patients quality of life. In recent years, there has been increased attention to Acceptance and Commitment Therapy (ACT) for cancer patients. Compared with other psychological interventions, ACT is less focused on symptoms of psychopathology and pays more attention to acceptance and values as influences on cancer patients' emotions, thus better addressing the idiosyncratic and dynamic nature of cancer adjustment. This study aimed to investigate the effectiveness of ACT on anxiety reduction in cancer patients.

Subjects and Method: A systematic literature search was conducted in multiple databases including PubMed, Science Direct, Google Scholar, Springerlink, and complemented by cross-referencing to identify randomized control trial published from 2016 to 2021. The following search terms were used: "Acceptance and Commitment Therapy" OR "Anxiety disorder" OR "Distress" OR "Cancer survivors". The inclusion criteria were English full text and randomized controlled trial. The articles were filtered using PICO model, including: (1) Population= cancer patients, (2) intervention= acceptance and commitment therapy, (3) comparison= non acceptance and commitment therapy, and (4) outcome= anxiety. The inclusion criteria were English full-text and reported mean and standard deviation. The study instrument for assessing anxiety was the Acceptance and Action Questionnaire II (AAQ-II). The systematic review was carried out according to the PRISMA guidelines. Data analysis were performed using RevMan 5.3.

Results: A meta-analysis involved 761 cancer patients divided into two groups, (1) 379 patients in the ACT group, and 382 patients in the non-ACT group. This study showed that acceptance and commitment therapy lowered anxiety in cancer patients, but it was statistically non-significant (Standardized mean difference $=-0.19 ; 95 \% \mathrm{CI}=-0.61$ to $0.23 ; \mathrm{p}=0.370$ ).
\end{abstract}

Conclusion: Acceptance and commitment therapy lower anxiety in cancer patients, but it was statistically non-significant.

Keywords: acceptance and commitment therapy, anxiety, cancer patients

\section{Correspondence:}

Prajna Pramitha Purba. Masters Program in Public Health, Universitas Sebelas Maret. Jl. Ir. Sutami 36A, Surakarta 57126, Central Java. Email: pramitha.purba@gmail.com Mobile: 082332787519. 